\title{
EXPERIÊNCIAS DE ENSINO-APRENDIZAGEM DE ESTUDANTES DE ENFERMAGEM EM UMA COMUNIDADE DO MUNICÍPIO DO RIO DE J ANEIRO
}

Experiences of the teaching-learning and experiences of students in nursing in a low income community of the city of Rio de J aneiro

Experiencias de enseñanza aprendizaje de estudiantes en enfermería en una comunidad de baja renta de la ciudad de Rio de J aneiro

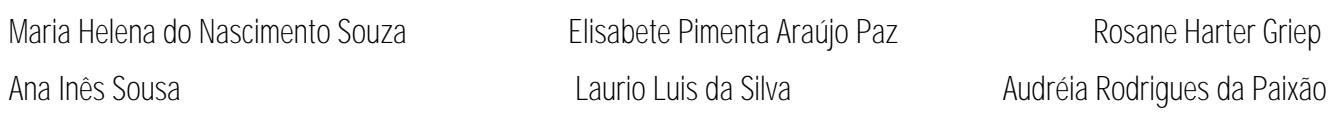

\section{Resumo}

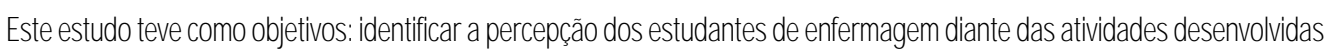
por les junto à Comnicadeedisatir o significado das atividades realizadas pelos acadênicos para a suaformação profissional Litilzarsea abordagemqualitativa, eaanostrafoi constitúda por 89 estudantes. Navoz dos mesmos, as

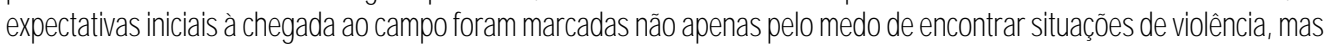

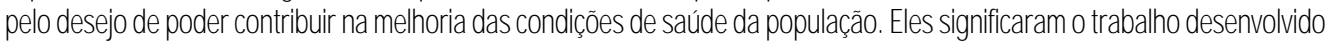
nacomeridadeconm possibilidadedecresaimento pessod, naior compreensão ca atu ação profissional do erfermeiroe

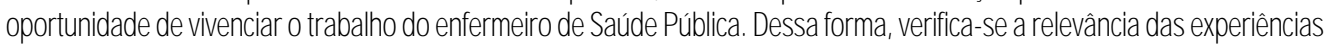
deensino-aprendzagememcom ridades para oprocesso deformação profissional dbsacadênicos deenfermagem

\section{Palavasdave}

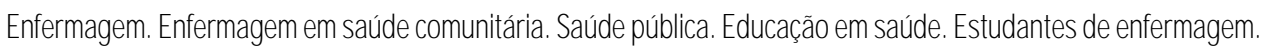

\section{Abstract}

Thisstudyithadas dijectivetoidentifytheperception of the students beforetheworkdendoped with thecommenity, and to arguemeaning of the adivities carried throughfor the academics for its professiond formation It twes usedqualitative boardingandthesampleves constituted by89students. In the vice of the same ones, theinitial expectations to the a rival tothefield hadbeennarked notonly bythefer to find violencesituations but bythe desire of being able to contributein theimproverent of theconditions of health of thepopulation Theyhadmeant work the developedinthe commuity as possibility of personal growth, bigger understanding of theprofessional performance of thenurse and chanceto livedeeply the work of then urse of Public Hedth ofthisformitisverifiedrelevenceof theexperiences of the teading-leaminginthecommenityfor theprocess of professional formation of thenursing academics.

\section{Resumen}

Esteestudio tuno como objetivo identificar la percepción de los estudiantes frenteal trabajo desarrolladb en una comnidad, y disatir el significado de las adtividades realizadas por los académicos para su formadión profesional. Seutilizó el abordajecualitativo yla mestra fieconstituidapor 89 estudantes. Enlavoz delos mismos, las expectativas iniciales a la llegada al campo fueron marcadas no sólo por el miedo de encontrar situaciones deviolendia pero por el deseo de poder contribuir enla mejoríadelas condiciones desaluddela poldación. Elas significarone trabajo desarrolladb enla comridadcomo posibilidad decreaimiento personal, mayor comprensión delaactuacón profesional del enfermeroyoportu ridad devivir el trabajo del enfermero deSalud Pública. Deesa forma, se verifica la relevancia de las experiencias de enseñarza aprendizajeenla com nidad para el proceso deformacón profesional delos acadámicos deerfermería.

Palabras dave Enfemeńa. Enfermería emsalud Keymords:Nursing Commenityheathrursing Pudicheath Healthedication Studats, nursing. comritaria Sálud pública. Eduradónensalud. Estudartes deerfermería 
INTRODUÇÃO

A atuação dos enfermeiros em comunidades constitui umcontraponto ao modelo assistencial que ainda predonina na naioria dos senviços de saúde do Pás, uma vez que este privilegia o atendimento dínico, individual, ancorado no pronto atendimento, na medicalização e na teonologia médico-hospitalar'. Tal modelo tem deixado mito a desejar, no que diz respeito à efetividade de soluções dos problemas básicos de saúde da população, prinaipalmente em locais nos quais o desemprego, a falta de moradia digna, a desigualdade social e a iniqüidade podem determinar a maior parte dos agravos relacionados à saúde das pessoas(Mbnteiro, Ferrianii).

A Enfermagem mestro apresentando avanços no campo social, ainda traz umolhar mensurador sobre as questões de saúde, com ênfase nos aspectos institurionais e instru mentais do seu fazer. Assim fazse necessário que os profissionais de enfermagem possamconsiderar as questões de anho socioattural, evidendadas numcontato mais direto entre eles e sua dientela, comb fatores condicionantes ao sucesso das suas intervenções, bemcomo dos autros profissionais de saúde(Dalmolin, Lopes, Vasconselos ${ }^{2}$ ). Desta forma, tais questões podempossibilitar aos profissionais, como umtodb, uma melhor compreensão da realidade sodial e de saúde da população atendida.

É comesse pensarento que pensantos ser possivel aos estudantes e docentes, no processo ensinoaprendizado, conhecer como vivem populações de baixa renda, seu local de moradia, sua histónia e sues redes sodiais. Isto favorece aos estudantes o contato direto coma realidade de vida dos usuánios dos senviços de saúde, o que gera condições de um aprendzadb condizente coma situação de vica e saúde da populaçãa. Adormo e Castro3 salienta que este olhar para as condições de vida das pessoas nos leva a querer conhecer o modo comb estas se articulame lidamcomo processo de adbecimento. Este tipo de conhecimento traduz-se em uma experiência intersubjeiva que conduz à revisão do sentido dado às coisas, podendo corresponder à necessicade de se situar e ter umespaço espeáfico na miltiplicidade de espaços presentes na contemporaneidade fragmentada $3: 34$

Com a intenção de fazer as aproximações necessárias entre ensino, pesquisa e extensão, o Departamento de Enfermagem de Saúde Pública da Escola de EnfermagemAnna Nery da Universidade Federal do Rio de Janeiro, através de seus docentes, desenvolve parte de suas atividades na Comunidade Mbro dos Cabritos, situada emCopacabana, Mriápio do Rio de Janeiro. Tratase de una comnidade onde, alémcla violência, do desemprego e das condições de pobreza, evidenciados daramente pelas precárias condições de vida de grande parte dos moradores, há pessoas que reconhecemo valor das ações voltadas para a prevenção de agravos e promoção da saúde.

Na abordagem das questões de violência urbana, e considarando o papel social da universicade, a opção da equipe empropiciar aos estudantes a vivênia neste tipo de cenário deriva da convicção de que a universidade deve formar não apenas enfermeiros, mas cidadãos comprometidos coma realidade social do pás, de nodo a contribuir coma melhoria da saúde e bermestar da população.

A inserção dos estudantes inscritos no Programa Curricular Interdepartamental IV (PO IV): "A Enfermageme os Cuidados Básicos de Saúde", nesta Comunidade, se dá com o desenvolvimento das seguintes atividades, dentre outras: levantamento socioeconômico, ambiental e de saúde das famílias residentes na comunidade; visitas domiciliares; elaboração de plano de assistência de enfermagem às famílias que apresentam problemas de saúde; avaliação e acomparhamento da situação de saúde e nutrição de crianças e adblescentes do Centro Educacional Cantinho da Natureza e da Creche Tia Sonia; consulta de enfermagemà criança comuso da estratégia de Atenção Integrada às Doenças Prevalentes na Infância (ADPI); identificação da prevalência de parasitose intestinal entre as crianças; orientações de saúde aos pais/responsáveis e funcionánios das creches; arsos de capacitação para educadoras e cozinheiras; elaboração de materiais educativos; atendimento aos idosos, realização de feiras de saúde na Comuridade; encaminhamentos para os seniços de saúde locais; elaboração de relatónios de atividades e de trabalhos científicos e trabalhos de Condusão de Curso. Alémdestas formas de atuação, alguns estudantes, após o período de estágio, solicitam a participação em ações extensionistas como voluntários de projetos de extensão ou ematividades coma comnidade

Comvistas a divilgar a experiência arrialar de discentes no desenvolvimento de atividades em comunidade, os objetivos deste trabalho foram a) identificar a percepção dos estudantes diante das atividades desenvolvidas por eles junto à Comnicade, e b) discutir o significado das atividades realizadas pelos acadêmicos para a sua formação profissional. 


\section{PROCEDIMENTOS METODOLÓGICOS}

Esta investigação sobre as experiências dos estudantes em trabalhos com comunidade é de natureza qualitativa e de caráter descritivo. A população deste estudo foi constituída por 115 estudantes que desenvolveramo Requisito Currialar Suplementar: Diagnóstico Simplificado de Saúde IV do Programa Ci mialar Interdepartamenta IV, na Comridade Morro dos Cabritos, no períodb de 2002 a 2004. Os sujetos foram89 estudantes deste grupo que concordaram emparticipar da pesquisa, o que representou $78 \%$ do total da população do estudb.

Para a identificação dos estudantes, uilizaramse a lista de inscritos no Programa e as escalas de estágio na Comunidade Morro dos Cabritos, no recorte temporal citado. Na ocasião do contato com os estudantes, obedecendo aos aspectos éticos, os pesquisadores apresentaramos objeivos do estudo e solicitarama participação voluntária dos mesmos, mediante assinatura do Termo de Consentimento Live e Esdaredido, conforme preconiza a Resolução 196/ 96 do Conselho Nacional de Saúdet . Utilizourse um questionário autopreenchido, semi-estruturado, contendo questões sobre experiênazs anteriores em comnidades, expectatives do primero contato com a comunidade, atividades realizadas no período do estágio, aspectos considerados como problemes de Saúde Pública e significados do trabalho na comnidade No preendhimento do questionário, foi esclarecido que seria garantido o anonimato dos perticipantes. Os instru mertos foramidentificados por duas letras, caraderizando o Programa arsado e o estudante A perda amostral foi de $22 \%$ caraderizada pela não-devolução do instrumetto.

A análise das respostas se deu após a orgarização das nesmas emquadros, a fimde se obter una maior ordenação das questões investigadas, composterior agrupamento dos temas de maior freqüência e formação de categorias temáticas (Mnayo5).

\section{RESULTADOS E DISCUSSÃO}

$\mathrm{Na}$ entrevista comos estudantes, buscamos saber se eles haviamtido contato comalguma comnidade antes da atuação deles na comunidade Morro dos Cabritos. Cerca de $70 \%$ deles não haviamido a qualquer comnidade Ao analisarmos as respostas sobre as expedatives dos estudantes no primeiro dia do trabalho na comridade, estas foramagnupadas em duas categorias: o medo de estar em uma comuridade violenta e o desejo de contribuir para a melhoria das condições de saúde da população.
O medo de estar em uma comunidade violenta

Observandb os relatos abaio, verificase que a grande expectativa dos estudantes era de vivenaiar situações de violênia social, as quais Ihes causavamreceio, medo e ansiedade, como expresso nas seguintes falas:

(...) Eu estava comreceio da violênaia e também não sabia como seria recebica. (AG)

A expectativa não era tão boa. Eu acreditava que teríamos dificuldade de acessar os dientes devido ao tráfico e que rão seríamos bemrecebidos. (AD) Por conta da violência que afeta grande parte do Rio de Janeiro, fiquei apreensiva quanto à possibilidade de sofrer algumtipo de violênaia (EO)

Eu tima a expectativa de encontrar mitas pessoas amadas e vendendo drogas. (AN)

Pelas respostas, fica daro que os estudantes, emum pineiro nomento, tirhammedb de desennolver atividades na comridade devidb ao receio de encontrar situações de vidênia geradas, dertre a tras formas, pelo tráfico de droges. A vidênda éde fato umgrande prodlenæ de Saúde Pública presente nos grandes centros urbanos, principal mente emrealidades onde a desigualdade social é mais evidente Entre os fatores determinantes da vidência encontramase o desemprego, a exdusão social e as condições inadequadas da vida urbana, e estes se constiturememimportantes desafios para os formiladores de políticas pablicas (Silva).

Sabe-se que situações de violência social podem constituir-se ainda emum problema para a atuação de profissionais ou acadêmicos da área da saúde que desenvolvemsuas atividades emlocais de maior risco, como o cenánio das comridades de baixa renda. Desta forma, ao realizar trabalhos coma população residente na comricade Morro dos Caloritos, a equipe reconhece a necessidade da tomada de certas medidas de segurança corro: contato com as lideranças, estabeleamento de parcerias comos atores sociais envolvidos, apoio de rentoros da comniclade, uso de uniforme e crachá e divulgação das atividades planejadas. Tais medidas têmfavoredido a permanêndia de docentes e estudantes nesta comunidade e possibilitado o estabeledimento de condições favoráveis ao processo ensino-aprendizagem

Contribuição para a melhoria das condições de saúde da população

Um outro aspecto relatado pelos estudantes nas respostas ao questionánio, emrelação às expectatives de iniciar o trabdho, fai o fato deesterepresentar a possibilicade de realizar ações voltadas à mellhoria das condições de saúde da população, conforme os seguintes relatos: 
(...) Poder prestar assistênja de Enfermagemde acordb coma necessickde, prinapalmerte às crianças. (DA)

(...) Poder cortribuir umpaquirho pera nethorar a saíde dos noradres da conmidade (BD)

(...) Patiajper des atividades que competemad enfermerira Tirha sensação de que era importate a mirha atueção ravida ddes. (EO)

Mrha expectativa era de encontrar una população que estivesse precisando da minha atuação e do reu grupo. (AG)

Desenvolver o conteúdo teórico de saúde pública na prática (AD)

É como tipo de pensamento voltado para melhorar as condições de saúde da população que os estudantes desenvolvernsuas atividades na comunidade. Este "ideário dos estudantes" vai ao encontro das propostas do Mnistério da Saúde, que na implementação de modelos assistendais, tais como o "Programa Saúde da Familia", recomenda que os profissionais de saúde realizem ações de proteção e promoção à saúde, ampliandb o foco do indvído para familia e comridade, comvistas à melhoria da qualidade de vida e nẽo apenes à reauperação da dbença(Mnayo7, Chiese et $\mathrm{a}^{8}$ ).

De acordo comPaaz, Souza e Giep', a inserção precoce dos conteúdos sobre o Programa Saúde da Família na grade curricular dos acadêmicos de enfermagem representa uma estratégia facilitadora para a formação profissional dos mestros. Nesse sentido, é importante que os estudantes percebam desde a sua formação, os nexos entre o ensino teórico e a prática assistendial, o que Ihes possibilitamelaborar idéias mais ampliadas dos significados de saúde para a população e quais seriamas suas responsabilidades nos vários níveis de atenção à saúde da com nidade.

Nesta perspectiva, Chirelli e Mskina ${ }^{10}$ ressaltamque, na formação dos acadêmicos da área da saúde, deve se ter a preou pação de constnir umprocesso attialadb como mundo do trabalho. Essa articulação ajuda a diminir as distânaias da relação teoria/prática e permite a uilização de nútodos mais ativos no processo ensinoaprendizagem desdobrando-se em aprendizagem significativa e mudanças nos diversos sujetos envolvidos no processo como umtodb. Isto determina a formação de profissionais mais unticos, reflevivos, comprometidos socialmente Dessa maneira, eles são sujeitos ativos e copazes de deliberações a respeito de si próprios, com desdobramentos no trabalho, contribuindo mais efeivarmente para a saúde da população.

Emvista dessa assertiva, e como desenvolvimento do trabalho na comunidade, os estudantes descreveram sua participação no PO. Pudemos destacar nas respostas deles duas categorias: crescimento pessoal e profissional e oporturidade de vivenciar o trabalho do enfermero de saúde pública

Crescimento pessoal e profissional

Esta categoria destaca a mudança que aconteceu na vida dos acadêmicos ao longo do desenvolvimento do trabalho na commidade, evidendiandb a superação das expectativas negativas e a possibilidade de maior compreensão da atuação profissional do enfermeiro, como exemplificado nos seguintes relatos:

Una ótima experiência, pois o aprendizado foi imenso, (...) conro pessoa e futuro profissional. (AM)

Significou aprendizado não só das questões a rialares, nas tambémde vida, dhar comautros olhos a comnidade; nemtodbs que estão lá são 'bandidos'. (AT)

Umnovo aprendizado, comvivências a respeito das condições sociais, e como orientar o autoaidado. (AD)

Meu cresaimento como pessoa, passando a ver o mundb a minha volta comumoutro olhar e meu cresaimento como futuro profissional. (AX)

Umaprendzado a mais. Comeste tipo de trabalho você percebe o quanto as pessoas necessitam de você (...). Foi importante e válido para minha vida profissional. (BA)

Significou reconhecimento da profissão, reconhedimento pessoal, liberclade de ação. (B)

Disatindo a relação entre espaço e aprendizagem na formação de profissionais de saúde, Fagundes ${ }^{11}$ verificou que as experiênias comcomuridades trazem aprendzagemsignificativa para os sujeitos, contribuindb para a reflexão acerca das formas tradicionais que as universidades concebemos a míalos ${ }^{11}$. Neste estudb, os estudantes referiramter adquiridb diversos tipos de saberes (aprendzagens) conv: 1) methor capadicade de comericação (falar e esatar); 2) aprender a se relacionar e a acolher o atro; 3) perceber que a população tem seus próprios saberes; 4) gerir situaçães que ocorrem no cotidiano do trabalho; 5) resolver problemas ciativamente; 6) atentar para unæ prática profissiona humarizada e 7) compreender de forma mais ampla os limites e as possibilidades da atuação profissiona. Estes são aprendizados que, na maionia das vezes, não são previstos nos objeivos das disciplinas, o que sugere pouca atenção por parte dos professores, ainda que os estudantes enfatizem essa necessidade. Desta forma, ressalta-se a importância de a universidade 
instituir "esatas" das práticas desenvolvidas e investir numa formação altura sólida e oútica dos estudantes.

Corroborandb esta posição, Toralles-Pereira aponta a preoaupação que a universidade deve ter na formação profissional no que diz respeito à competêndia técrica associada ao aspecto ańtico e à capaciclade de resolver proderis, trabalhar emequipe e assumir responsabilidades, participando de forma mais ativa do processo de aprenotzagemede modança dos syjetos. Acerca dsso, cabe ressatter que a caraterística a mialar de grad enção da Escola de EnfermagemAma Nery temcono prerogativa que o ensino aconteça da prática pera a teoria o que, de certa fơma faz comque o aprendzadb seja dinânico e beseadb ra prodlendização, a partir da prática assistendal.

Neste sentido, segundo Chirelli Mskima ${ }^{10}$ as mudanças sociais têmpossibilidade de ocorrer na medida emque os profissionais possamaturar com sujeitos do processo de atenção à saúde, construindo umcontexto onde as relações interpessoais realizam se numa relação mais democática, compossibilidade de crescimento dos sujeitos participantes de um projeto solidánio, na direção de acõos que busquema emancipação do usuário e da comuidade

Oportunidade de vivenciar o trabalho do enfermeiro de Saúde Pública

Quando as perguntas do questionário versarama respeito dos significados acerca da experiênia dos estudantes de atuaremna comnidade, comvistas a vivenciaremmais de perto o trabalho do enfermeiro de Saúde Pública, os mesmos responderam

Una experiênaia única como trabalho emSaúde Pública, onde me senti útil para a comricade (...) foi una vivência enorme. (AF)

\section{Anoção verdadara do trabalho emSádePúdica(BD)}

Me ajudou a definir o papel do enfermeiro dentro de una comricade (B)

Fa umtrabalho mito bomque me fez visualizar a importância da Saúde Pública, do trabalho em comnicades carentes que necessitam de nossa intervenção (DE)

Significou a tentativa do entendimento dos problemas básicos que acontemesta população (DO)

Alémde a maioria estudantes apontar a satisfação diante do desenvolvimento das ações na comridade, evidenciadas pelas respostas referentes à percepção de cresaimento pessoal e profissional, tambémfoi referida a atuação do enfermeiro de Saúde Pública de forma ampliada, mais autônoma, uma vez que o trabalho nas comunidades requer maior autonomia profissional para a promoção e a proteção da saúde dos indvíduos e famlias. Nesse anbiente, o enfermeiro pode realizar importantes ações de saúde junto aos diversos grupos humanos, a partir da compreensão das condições nas quais a população vive, contribuindo mais efetivamente para a melhoria da qualidade de vida e da saúde da população(Silva e Tanaka ${ }^{13}$ ). Pensamos que a atitude dermonstrada pelos estudantes foi possível gracas ao fato de, junto comeles, termos estabeledido estratégias de intenenção na comnidade que dziamrespéto à prevenção de agravos e pronoção da saúde, bemcomo as ações arativas necessánias às situações encontradas. Isto favoreceu o constante aprendizado das práticas de saúde coletiva e mais, a aproximação dos estudantes comas ciranstânias de praticar ações de enfermagem nas comunidades, a partir de um diagnóstico de saúde elaborado e implementado por eles, comsupenisão docente.

De acordo com Vaughan e Morrow ${ }^{4}$, em saúde comvitária, os diagnósticos de saúde da comridade subsidiam a decisão de implantar programes govemamentas mais efetivos para melhorar a situaçã de saúde da população. Da mestra forma, Fonseca e Bertolozzi ${ }^{15}$ mencionamque a identificação dos prodlemæs de saúde da comeridade pode ser útil no que dz respecto à disaussão e implenertação de propostas de intenencões que busquemtransformar o conteúdb das práticas sociais na saúde, bemcorro suas atiaulações na sociedade ou emgrupos especáficos.

Segundo Silva e Tanaka13, as pessoas na comunidade são reconhedidas como os sujeitos privilegiados de atuação do estudante no processo saúde-doença, fato que propicia a ampliação da visão da dbença presente na effera hospitalar.

Para tanto, ra atenção à saúde da população, delese lever emconsidaração a redidade social, os estilos de vidae as necessidades emsaíde, caraderísticas que dfialmerte são identificadas ra sua totalidade, no antierte dos servicos de saúde Tathez por isso, Sika e Tanda' dimemque as comeridades são recorhedidanerte u mespasco privilegiadb para a a tueção dos estudartes. Para démdsso, aímanos que ensiner nes comeridades é un exclete oparturidade para ampliar a visão de mendo dos estudantes.

Neste serticb, a presença dos estudartes no espaço da comeridade propicia naior sensibilidade emrelação aos prodlemes de saúde da população, naior qualificação pelas ações prestadas e o estabeleaimento de una relação de parceria comos senviços que posiciona os professores e etudantes conø atores e coresponsádes, emútinm instânà,

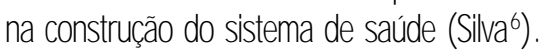




\section{CONCLUSÃO}

A Escola de EnfermagemArna Nery, através dos docentes do Departamento de Enfermagem de Saúde Pública, tempropiciado aos estudantes de graduação experiências junto às comunidades do muniápio do Rio de Janeiro. Nelas, os estudantes desenvolvemações de pesquisa, ensino e extensão, possibilitando a adequação dos conhecimentos teóricos à prática e, conseqüentemente, o desenvolvimento da capadidade de participar mais ativamente do processo ensino-aprendizagem

Apesar da relevância desta prática para a aprendizagem dos conteúdos de Saúde Pública, verificase que, mitas vezes, o estágio a rrialar em comunidades não é implementado nos aursos de graduação emvirtude de vários fatores, como medo de sofrer violêndia (espedialmente nas comnidades de baixa renda em grandes centros urbanos), desconhecimento da metodologia adequada para este tipo de trabalho, falta de interesse de docentes e discentes, influência do paradigma biologicista hospitalocêntrico, alémca facilicade ematender apenes os indivíduos que comparecemnos serviços de saúde e a conseqüente falta de disponibilidade para ir ao encontro do autro emseu contexto e problenática.

A experiência do trabalho na comridade, para os estudantes de enfermagem que realizaram o Dagnóstico Simplificado de Saúde IV, foi considerada essendal para a aquisição de competênias esperadas para sua formação profissional, sendo possível obsenvar a assodiação das experiências práticas ao conhecimento teórico e crítico do processo de enfermagem na atenção à saúde dos indivíduos e famílias, independenterente das pressões cotidianas

\section{Referências}

1 MurteiroAL, FeriariMGCAtençãoàsádedaciança pesspetinadapática

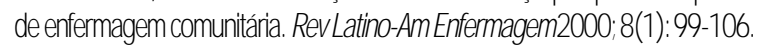

2DanndinBMLopesSMB, VesconcellosMPCAconsturçãomedodógica dbcampo etnografia criatividadeesensibilidadenain nestigaçãa. Saúde eSoc[online] 2002; [ätadb ]11(2), Lisponívedentttp//apsp.org.br/ saudesodiedada/X 2/construca_ nedodogicaltm

3.AddrmoRCF, GastroAL Oeeráciodasenibibilidade pesquisaq alitativa easaúdeconoqualidade SadeSoc 1994; 3: 33-42.

4. ConselhoNkaiond deSaúde Dretrizesenomæs regulamentadoras depesquisasemsereshumanos. Resoluçãon 196de10deautubro de 1996. In: OMndodaSaúde1997; 21 (21).

5. Mnayo MCS Odesafio db conheamento: pesquisaqualitativaem saúde RiodeJaneiro(B)). HUTECABRASCO, 1998. que narcama trajecónia de vida e da saúde da maioria das pessoas que residememcomnidades.

Neste contexto, os estudartes puderamter consciênda do seu papel social, foramcapazes de cturar na promoção, proteção e reauperação da saúde de pessoas e realizar atividades de pesquisa acerca dos problemas energentes no antiente da comridade Há que se dosenvar que quando desennolvemessas atividades na comridade, os estudantes iniciamumcontato mais fre qüente com "as realidades socias", o que pode significar una disaussão prơfía para a solidficação de ummode lo interdisajpliner de atuação para a área da saúde.

Na voz dos estudantes, as expectatives iniciais por ocasião da chegada ao campo forammarcadas não ape nas por nedo de encontrar situações de violêndia, nas pelo desejo de contribuir para a melhoria das condições de saúde da população. Essas expectatives significaramo trabalho desenvividb na comridade como possibilida de de cresaimento pessoal, naior compreensão da atua ção profissional do effermeiro e oportu ridade de vivenciar o trabalho do enfermero de Saúde Púdica.

Condui-se que as experiênias proporcionadas aos estudantes de enfermagemna comunidade não se restringiramapenas emumaumento de conhedimentos técicos, mas refletiramtanbémo amadureaimento pessoal dos mesmos durante todo o estágio, ampliando sua compreensão do que seja o trabalho de Saúde Pública, do valor da pessoa que é aidada ou que está disposta a receber aidados e da possibilidade de troca de saberes. Nesta perspectiva, a interação dos estudantes coma população no seu contexto social, assimcomo a cooperação estudantedocente mostraramse importantes para o processo de ensino-aprendizageme para o desenvolvimento da autonoma profissional dos futuros enfermeros.

6. SlvaSF. Cresaimentodavidêniaurbana as grandes cidadesestão dartede una epidemiasocia? DuulgSaudeDabate2004; 3: 10-14.

7. MristériodaSáde(BR). SaúdedafanllianoBrasil: limesestraté gicas paraoquadiêiio1999/2002. Braslia(DF): SecretariadePolíticas deSaúde/ Departarento deAtençãoBásica

8. CiesaAM LislaineAF. Otrdbalhodos agentes comnitáios desaúde resgrandescidades análisedbseuptendial naperspectivadapronoção dasaúde RevBrasSádedaFamilia 2004; 5(7): $42-50$.

9. PazEA SouzaMHN GiepRH Programasesaúdedafamília: experiênias deensino eatuação degraduandos deerfermagem EscArna Nery RevEnferm2003; 7(3): 439-44.

10. Cirelli MQMSkimaSM Aformaçaodberfermerocútico-relexio no arso de enfernagemda Faculdade de Medicina de MańliaFAMEMA Revlatino-AmEnfermagem2003; 11(5): $574-84$ 
11. Fagundes NC, BurmhamiF. Disatindbarelaçãoentreespaçoe aprendizagenna formação deprofissionais de saíde. I Interface 10 mricação, saúde, edração 2005; 9 (16): 105-14

12. Toralles-PereiraM Nbtas sobreeducacãon natransição para umnovo paradigma. Interface comnicação, saúde, educação 1997; 1(1): 51-8.

13. SivaPF, TandaOY. Aprendzagembeseedanacomericade una experiência na formação de profissionais desaúde. OhoMágico 2004; 11(4): 3-12.

14. VaughanJP, MarrowRH Epidamiologia para mnicápios. São Paulo(S). HUTाEC, 1997

15.Fonseca RMGS, Bertolozi MR Aepideniologiasocial eazssistênciaásaúdeda população. ABEn, 1997. (SérieDidáticaErfermagemnoSS).

\section{Sobre os Autores}

\section{MiralteradoNiscineloSoura}

Datoraen Efertagem,ProfAssistertedb DepatanertodeE Eremagem

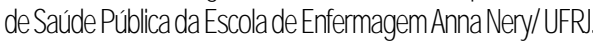

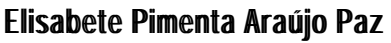

DatoraemEnfermagem, Profe-Aduntadb Depto. deE fermagemde SaúdePúdicadaEscoladeE EfermagemAmaNery/ URI.

\section{RosaneltaterGiep}

DoutoraemCiências, Prof̣̂Adunta do Depto. deErfermagemde SaúdePúdicadaEscoladeEffermagemAmaNery/URI.

\section{Arahn̂ิsSousa}

DatoraemCiênias daSaúdd/-SaúdeclaMulher, PrợeAdunta do Depto. deE EfermagemdeSaúdePúdica daEscoladeEtermagem Amankery URI.

\section{Laviolu'sdastra}

Espedialista em Enfermagemdo Trabalho. EnfermerrodaPrefeitura deNbvalguagu/Riodejaneiro.

\section{AndéaRodiguesdapaixäo}

Acadênicadb 80períodb db Curso degrad accão deE Efermagemda EscoladeEfremagemAmaNery/URI. 